\title{
Patient Acceptance and the Psychological Effects of Women Experiencing Telecolposcopy and Colposcopy
}

\author{
Daron G. Ferris, MD, Mark S. Litaker, PhD, Priscilla Ann Gilman, MD, and \\ Abidee G. Leyva Lopez, MHS
}

Background: The purpose of the study was to assess patient acceptance and psychological effects in women experiencing telecolposcopy compared with colposcopy.

Methods: Convenience samples of 150 and 263 women scheduled for colposcopy or telecolposcopy, respectively, completed questionnaires assessing anxiety (Prime MD), depression [Center for Epidemiologic Studies/Depressed Mood Scale (CES-D)], health beliefs and concerns, coping style (Miller Behavioral Style Score) and examination acceptance and satisfaction. Test scores and subject responses were compared using the $t$ test and Wilcoxon rank sum test.

Results: Mean scores representing mild anxiety $(1.3$ and $1.2, P=.7)$ and mild depression $(35.4$ and $36.3, P=.4)$ were reported for the telecolposcopy and colposcopy groups, respectively. The telecolposcopy group indicated significantly greater mean scores for the examination, saving them time and money compared with the colposcopy group. Women in both groups were highly satisfied with their examinations and care.

Conclusions: In general, women reported very high levels of satisfaction with telecolposcopy and colposcopy. Potential savings of time and money and improved health care were considered of particular value to women examined by telecolposcopy. Telecolposcopy seems to be well accepted by rural women. (J Am Board Fam Pract 2003;16:405-11.)

Telecolposcopy enables the examination of women's lower genital tract by experts located at a distant site. ${ }^{1}$ Such a service provides immediate and necessary consultation for approximately one third of women examined by rural primary care colposcopists. ${ }^{1}$ Thus, expensive travel is obviated for many rural women who cannot afford transportation costs and consequently fail to have proper evaluation by a consultant at a distant medical center. Sophisticated or simple telemedicine systems provide either real-time (video-like) colposcopic images or static (store-and-forward) digitized col-

Submitted, revised, 13 January 2003.

From the Gynecologic Cancer Prevention Center (DGF, MSL), the Departments of Family Medicine (DGF), and Obstetrics and Gynecology (DGF), the Office of Biostatistics and Bioinformatics (MSL), and Department of Psychiatry and Health Behavior (PAG), The Medical College of Georgia, Augusta; and the Instituto Nacional de Salud Publica, Cuernavaca, Mexico (AGLL). Address correspondence to Daron G. Ferris, MD, Medical College of Georgia, 1423 Harper Street, HH-105, Augusta, Georgia 30912-3500 (e-mail dferris@mail.mcg.edu).

This study was supported in part by a grant from the Agency for Health Care Policy and Research, and the National Cancer Institute, National Institutes of Health, Bethesda, Maryland. pophotographs for assessment purposes. ${ }^{2,3}$ Videoadapted colposcopes operated by a local colposcopist are used to capture then transmit these images through broadband fiber optic or standard telephone lines. ${ }^{4}$ Compatible supporting electronic and/or computer equipment located at the transmitting and receiving sites are necessary for clinician-to-clinician interaction and consultation. Some high-speed systems allow synchronous faceto-face communications between distant experts and patients.

From the patient management perspective, the diagnostic accuracy of telecolposcopy is comparable with that delivered on site by local clinicians. ${ }^{1}$ Distant experts are able to support and direct less experienced colposcopists while women are evaluated locally. Hence, telecolposcopy may reduce the need for costly referral. ${ }^{1}$ Barriers to expert health care are consequently eliminated for those women unwilling or unable to travel for consultation. The provision of enhanced health care may be delivered by telecolposcopy with only small increases in cost. ${ }^{2}$

Although telecolposcopy seems feasible from the technical, financial, and patient management per- 
spective, the potential psychological repercussions are unknown. Women have been previously shown to experience certain adverse emotional responses to abnormal Papanicolaou smears and traditional colposcopy. ${ }^{5-17}$ How might women respond to having pictures of their genitalia sent to unknown health care personnel in another town? Modern technology will improve health care only if women accept and embrace the practice. The purpose of this study was to compare patient acceptance and the psychological effects women experience in association with telecolposcopy and colposcopy.

\section{Methods}

A convenience sample of women scheduled for a colposcopic examination at the Ware County Health Department, Waycross, GA, and the Tri County Clinic, Warrenton, GA, were asked to enroll in an Institutional Review Board (IRB)approved telecolposcopy study. Another convenience sample of women scheduled for a colposcopic examination at the Family Medicine Center or Comprehensive Cancer Center colposcopy clinics at the Medical College of Georgia, Augusta, GA, were also asked to enroll in a similar abbreviated study. All women read then signed an IRBapproved informed consent document before participating. Inclusion criteria included recent abnormal Papanicolaou smear, grossly abnormalappearing cervix, returning for surgery, or postsurgical follow-up examination. Exclusion criteria included excessive menstruation, acute cervicitis, pregnancy, lack of interest, lack of appropriate response to a written questionnaire, and transient telecolposcopy communication problems.

Demographic information, including age, marital status, education, and annual income, were obtained initially from both groups. Before their colposcopic or telecolposcopic examinations, subjects completed validated and commonly used questionnaires ${ }^{18}$ assessing anxiety, depression, coping styles, and health beliefs and concerns, as reported previously. ${ }^{19}$ Anxiety was assessed using an abbreviated version of PRIME-MD. ${ }^{20}$ Depression occurring the previous week was evaluated by the 20-item Center for Epidemiologic Studies/Depressed Mood Scale (CES-D). ${ }^{21}$ Two coping styles, monitoring and blunting, were assessed using the Miller Behavioral Style Scale (MBSS). ${ }^{22}$ High monitors attend to threatening situations, whereas blunters tend to avoid them. A 12-item questionnaire was used to assess health beliefs and concerns. ${ }^{23}$

Before being examined by telecolposcopy, women were told about the brief expert examination followed by the telecolposcopic examination. The details of the telecolposcopic examinations have been published previously. ${ }^{1,2}$ In brief, however, an on-site expert colposcopist performed an initial, blinded colposcopic examination that prohibited cervical biopsy or use of Lugol's iodine solution. Next, a rural (local) colposcopist (family physician or nurse practitioner) conducted a colposcopic examination at 1 of 2 rural satellite telemedicine clinics within the Medical College of Georgia Telemedicine System. Another expert colposcopist at the central telemedicine center monitored this examination. The distant expert provided limited verbal consultative assistance when requested, but the local colposcopists performed histologic sampling. Both real-time and store-and-forward telecolposcopic images were transmitted for each subject. Women examined at the central site had a traditional colposcopic examination that did not involve video recording or the transmission of colposcopic images. Expert colposcopists conducted these examinations.

After their colposcopic or telecolposcopic examinations, subjects completed a 23 -item questionnaire scored using a Likert scale $(1=$ strongly disagree to $5=$ strongly agree). The questions assessed various issues, including satisfaction with the examination, cervical cancer concerns, personal recognition by the consultants, examination preference, comfort, and potential cost/time savings of telecolposcopy. For questions to make sense for a particular colposcopic examination, some were slightly rephrased as appropriate.

Demographic statistics were calculated for each group. Mean values of continuous variables were compared between the groups using $t$ tests for variables with approximately normal sample distributions and Wilcoxon rank sum test for variables that were not normally distributed. The $\chi^{2}$ test was used to compare distributions of categorical variables between the groups. For tables containing expected counts of less than 5, the Fisher exact test was used. Scores for each of the survey scales were calculated by summing the values for each of the scale items. Scale scores were compared between groups by Wilcoxon rank sum test. Total scores on the mon- 
Table 1. Mean Health Beliefs and Concern Scores for Colposcopy and Telecolposcopy Subjects

\begin{tabular}{lccc}
\hline & & Cohorts & \\
\cline { 2 - 3 } Concern/Belief & Colposcopy & Telecolposcopy & $P$ Value* $^{*}$ \\
\hline Concern about disease & $3.3(1.1)$ & $3.3(1.1)$ & $\mathrm{NS}^{\dagger}$ \\
Concern about procedure & $3.2(1.4)$ & $3.4(1.4)$ & $\mathrm{NS}$ \\
Concern about consequences & $3.1(1.2)$ & $3.1(1.2)$ & $\mathrm{NS}$ \\
Importance of appointment & $3.6(0.7)$ & $3.6(0.8)$ & $\mathrm{NS}$ \\
Self-blame for disease & $2.9(1.3)$ & $2.8(1.4)$ & $\mathrm{NS}$ \\
Self-control over disease & $3.7(1.2)$ & $3.9(1.2)$ & $\mathrm{NS}$ \\
Doctor has control over disease & $4.1(0.9)$ & $4.2(1.1)$ & $\mathrm{NS}$ \\
\hline
\end{tabular}

Pre-examination scores reported using a Likert scale $(1=$ not at all and $5=$ extremely). Data are presented as mean (SD).

* Wilcoxon rank sum test.

† NS, not significant $(p>.05)$.

itor and blunter scales were categorized as greater than 11 or not, and the proportions of subjects in each category were compared between groups using the $\chi^{2}$ test.

\section{Results}

At the conclusion of the study, 263 women had been examined by telecolposcopy and 150 women by standard colposcopy. There was no significant difference ( $P=.41, t$ test) in subject age for the telecolposcopy and colposcopy cohorts $[31.5 \pm$ 13.2 (mean $\pm \mathrm{SD}$ ) and $32.6 \pm 12.2$, respectively]. There was also no significant difference in the highest level of education completed between the 2 groups. However, a significantly greater percentage of colposcopy subjects were single $(52.4 \%$ vs $38.5 \%)$ or indicated a "live in" relationship (7.4\% vs $3.9 \%$ ) compared with the telecolposcopy group, who reported greater rates of being married, divorced, widowed, or separated $\left(\chi^{2}, 15.4 ; P=.02\right)$. A significantly greater percentage of telecolposcopy subjects ( $43.6 \%$ vs $32.6 \%$ ) reported annual incomes less than $\$ 6000$ per year compared with the colposcopy subjects $\left(\chi^{2}, 9.5, P=.02\right)$. Although exact figures are unavailable, more than $95 \%$ of eligible rural women agreed to participate.

We assessed potential anxiety by using the pertinent questions from PRIME-MD. Mean scores for the telecolposcopy group $(1.26 \pm 1.1)$ and colposcopy group $(1.20 \pm 1.0)$ were not significantly different $(P=.7 ; t$ test $)$. Clinically, these mean scores would represent a mild level of anxiety.

Potential depression was assessed using the Clinical Epidemiologic Depression Scale-D. Scores within the range of 30 to 36 indicate mild depression. Telecolposcopy and colposcopy subjects had mean scores of 35.4 and 36.3, respectively. These scores were not significantly different $(P=$ .4; $t$ test).

Using a survey of 12 questions, we assessed health beliefs and concerns of subjects before their examinations (Table 1). However, there were no significant differences in mean responses between groups. In general, women in both groups were not excessively concerned about the procedure, disease, or consequences.

We also assessed 2 coping styles, monitoring and blunting, using 4 hypothetical situations (Table 2). The telecolposcopy group had a significantly greater mean monitor score (10.4) compared with the colposcopy group $(9.5 ; P=.03$, Wilcoxon rank sum test). There was no difference between mean blunter scores for the 2 groups. We also compared the proportion of women with high monitor and blunter scores $(>11)$ between the telecolposcopy

Table 2. Mean Monitor and Blunter Coping Style Scores for Colposcopy and Telecolposcopy Cohorts

\begin{tabular}{lccc}
\hline & & \multicolumn{2}{c}{ Mean Coping Style Score } \\
\cline { 3 - 4 } Cohort & $\mathrm{N}$ & Monitor & Blunter \\
\hline Colposcopy & 150 & $9.5(3.5)$ & $4.6(2.6)$ \\
Telecolposcopy & 263 & $10.4(3.5)^{*}$ & $4.8(2.7)^{\dagger}$ \\
\hline
\end{tabular}

Monitors attend to threatening cues; blunters avoid threatening cues. Scores $>11$ indicate high monitor or blunter, scores $<11$ indicate low monitor or blunter. Data are presented as mean (SD).

* Comparison of monitor scores between cohorts, $P=.03$, Wilcoxon rank sum test.

† Comparison of blunter scores between cohorts, $P=.6$, Wilcoxon rank sum test. 


\begin{tabular}{|c|c|c|c|}
\hline \multirow[b]{2}{*}{ Question } & \multicolumn{3}{|c|}{ Cohorts } \\
\hline & Colposcopy & Telecolposcopy & $P$ Value* \\
\hline Examination was painful & $2.3(1.2)$ & $3.0(1.3)$ & $<.001$ \\
\hline Regular clinician can find abnormalities & $3.1(1.3)$ & $3.5(1.2)$ & .005 \\
\hline Tele-/colposcopy examination saved me money & $3.6(1.0)$ & $4.1(1.0)$ & $<.001$ \\
\hline Not satisfied with examination & $1.7(1.1)$ & $1.9(1.3)$ & $\mathrm{NS}^{+}$ \\
\hline Tele-/colposcopy improved diagnostic accuracy & $4.4(0.8)$ & $4.4(0.8)$ & NS \\
\hline Getting cancer would be serious & $4.7(0.7)$ & $4.7(0.8)$ & NS \\
\hline Tele-/colposcopy improved quality of my care & $4.4(0.9)$ & $4.6(0.7)$ & NS \\
\hline Would not like name recognition by distant expert & $3.1(1.4)$ & $2.8(1.4)$ & .04 \\
\hline Telecolposcopy saved/would save me time & $4.0(1.0)$ & $4.3(0.9)$ & $<.001$ \\
\hline If clinician says telecolposcopy improves my care, I'll do it & $4.4(0.7)$ & $4.6(0.6)$ & $<.001$ \\
\hline $\begin{array}{l}\text { Thoughts and fears of cervical cancer after told about } \\
\text { abnormal Papanicolaou smear }\end{array}$ & $4.0(1.0)$ & $4.2(1.1)$ & .02 \\
\hline Difficult to travel 1 to 4 hours for medical care & $3.4(1.4)$ & $3.9(1.3)$ & $<.001$ \\
\hline $\begin{array}{l}\text { Distant expert can see/could have seen my cervix as } \\
\text { well as local clinician }\end{array}$ & $3.5(1.1)$ & $4.1(1.0)$ & $<.001$ \\
\hline Recommend tele-/colposcopy to a friend & $4.4(0.7)$ & $4.5(0.7)$ & NS \\
\hline Felt better about health after tele-/colposcopy examination & $4.3(0.9)$ & $4.4(0.8)$ & NS \\
\hline Felt comfortable with tele-/colposcopy exam today & $4.3(0.9)$ & $4.3(0.8)$ & NS \\
\hline Satisfied with tele-/colposcopy and care & $4.6(0.7)$ & $4.6(0.6)$ & NS \\
\hline Confident distant expert/colposcopist was competent & $4.6(0.7)$ & $4.6(0.6)$ & NS \\
\hline Felt distant expert/colposcopist concered about my health & $4.5(0.8)$ & $4.5(0.7)$ & NS \\
\hline
\end{tabular}

Postexamination scores reported using a Likert scale $(1=$ strongly disagree and $5=$ strongly agree). Some questions slightly rephrased as appropriate for each situation. Data are presented as mean (SD).

* Wilcoxon rank sum test.

† NS, not significant $(P>.05)$.

and colposcopy groups. A significantly greater percentage of the telecolposcopy group $(41.8 \%)$ were considered high monitors compared with the colposcopy group $\left(32.0 \% ; P=.04 ; \chi^{2}\right)$. There was no significant difference between the telecolposcopy and colposcopy groups in proportions of women considered high blunters $(2.3 \%$ and $0.7 \%$, respectively).

Subjects answered a set of 23 questions after the telecolposcopy or colposcopy examinations (Table 3 ). Of the 23 questions, significant differences in mean patient responses between the 2 groups were noted for 9. In comparison with the colposcopy group, women in the telecolposcopy group had greater mean scores with the examination saving them money and time. The same group also had a greater mean score concerning the difficulty of traveling 1 to 4 hours for medical care. The telecolposcopy group was less concerned about the distant colposcopist knowing their names. The colposcopy group was less convinced the distant colposcopist would be able see the cervix as clearly as the local clinician could. The telecolposcopy group was more likely to trust the colposcopic skill and the advice of their local clinician to have telecolposcopy because it would improve their care. The colposcopy group was less likely to have thoughts and fears about cancer, and reported less pain with the colposcopic examination. There were equivalent high levels of satisfaction and comfort with both types of examinations.

\section{Discussion}

Patient acceptance of new technology should ideally be considered before implementing a new health care system. This is particularly true when gynecologic examinations interject cameras transmitting potentially sensitive images to distant observers. We have previously reported the technical attributes and deficiencies of real-time telecolposcopy and computer-based telecolposcopy. ${ }^{1,2}$ Both may allow useful clinical consultative support for patients otherwise unlikely to benefit from expert oversight. Furthermore, computer-based telecol- 
poscopy may offer certain cost savings for women located in underserved areas. ${ }^{2}$ Unfortunately, federal and private payors have been slow to reimburse for telemedicine examinations. ${ }^{24}$ Although a novel instrument may improve health care delivery, it may not be readily adopted by patients, clinicians, or third-party payors.

This study has demonstrated that telecolposcopy is favorably accepted by women in underserved rural regions. These women had high comfort and satisfaction with telecolposcopy. In general, women thought telecolposcopy improved the quality of their care. They felt better about their health after the examination. Furthermore, as an indication of their approval, women would recommend it to a friend. Women also thought telecolposcopy saved them both time and money. The latter issue is critically important given the fact that more of the telecolposcopy patients lived in poverty. A lack of financial resources and significant travel distance for consultation both pose impediments to access.

Concerns about a previously unknown distant expert examining their genital region from afar seemed unwarranted. Women were very confident that the distant colposcopist was competent. This positive response may have resulted from the brief preliminary introduction by the local clinician or the simple assumption that practice at a medical university implies expertise. Women also felt the distant colposcopist was concerned about their health. This belief may have been derived by having a second monitor in the examination room devoted to transmitting a live image of the distant expert. However, not all telecolposcopy systems provide dual video linkage. Women were comfortable with the distant expert knowing their names. As such, maintaining anonymity is not perceived necessary. Perhaps name recognition helps to personalize the remote examination.

Of particular interest was the fact that women thought the distant clinician could see the cervix as clearly as the local clinician could. The quality of resolution for real-time and computer-based systems is inferior to that viewed through a colposcope. ${ }^{25}$ Yet this image, also observed by subjects on a second monitor, seemed of sufficient quality to untrained eyes.

Although expert colposcopists were involved in their care, rural women had greater trust in the colposcopic skills and advice of the local colposco- pist. This confidence may be partly a result of the long-standing continuity of care delivered to these rural women. Most women in the colposcopy group were referred by primary care clinicians for evaluation, whereas primary care providers examined the telecolposcopy group.

Women in the colposcopy group had fewer fears and thoughts of cancer compared with the telecolposcopy group. Whether level of education, preexamination counseling, or clinical demeanor influenced this difference is unknown. The colposcopy group also reported significantly less pain with the colposcopic examination. We are unsure of the explanation for this finding. Perhaps the slightly prolonged time required for the telecolposcopy component or additional manipulations for the distant colposcopist increased patient discomfort. Otherwise, clinician technique and dual examinations by the on-site expert and local colposcopist may have accentuated pain. In practice, however, telecolposcopy occurring outside a research study would not involve an additional on-site examination.

Both groups experienced mild anxiety and mild depression before their colposcopic appointments. These levels of anxiety and depression were equivalent to those observed by women before a primary care appointment. ${ }^{19}$ Therefore, it would seem that rural and urban women react similarly to hearing about the results of an abnormal Papanicolaou smear. Better education may help allay unnecessary fears and anxiety.

The telecolposcopy group had a significantly greater percentage of women considered to be high monitors. Why rural women might be more attentive and concerned about their health care needs is unknown. This same group was more likely to have greater thoughts and fears about cancer. Given these heightened concerns, it is reassuring that the rural women would be more likely to address the results of an abnormal Papanicolaou smear, as would be expected from high monitors. Greater patient compliance for a procedure many women would rather not undertake and improved access to consultative telecolposcopy are complementary factors poised to enhance the management of women with lower genital tract neoplasia.

This study is limited by the economic and feasibility restraints that permitted only 2 rural colposcopists and 2 sites in the trial. Women's responses may have varied if a greater number of 
rural sites and colposcopists were considered. However, the sites differed by racial profile, distance from the central telemedicine center (1-hour vs 4-hour drive), type of clinic (health department versus private clinic), and provider specialty. The potential number of sites was limited by the requirement that statewide telemedicine services exist on-site and that a trained local colposcopist be present. Some study findings (ie, pain experienced, quality of care) may have also been influenced by the necessity for 2 consecutive on-site colposcopic examinations by the telecolposcopy cohort. The alternative of rescheduling subjects for an expert examination later was considered awkward. The local clinician's biopsies would have altered the cervix for an expert examination later. Furthermore, noncompliance with a delayed expert examination would have severely limited the number of assessable subjects. Finally, although the 2 cohorts differed by marital status and income, other undocumented differences probably existed. It is unknown whether these unspecified differences could have influenced our findings.

\section{References}

1. Ferris DG, Macfee MS, Miller JA, Crawley D, Watson D. The efficacy of telecolposcopy compared with traditional colposcopy. Obstet Gynecol 2002; 99:248-54.

2. Ferris DG, Bishai DM, Macfee MS, Litaker MS, Dickman ED, Miller JA. Telemedicine network telecolposcopy compared with computer-based telecolposcopy. J Lower Genital Tract Dis. In press.

3. Ferris DG, Litaker MS, Macfee MS, Miller JA. Remote diagnosis of cervical neoplasia: 2 types of telecolposcopy compared with cervicography. J Fam Pract 2003;52:298-304.

4. Ferris DG, Willner WA, Ho JJ. Colpophotography systems: a review. J Fam Pract 1991;33:633-9.

5. Magherini P, Cecchini S, Catagni CF, Francisca FD, Ciatto S, Grazzini G. Emotional reactions and colposcopy. J Epidemiology Community Health 1996; 50:108-9.

6. Tomaino-Brunner C, Freda MC, Damus K, Runowicz CD. Can precolposcopy education increase knowledge and decrease anxiety? J Obstet Gynecol Neonatal Nurs 1998;27:636-45.

7. Freeman-Wang T, Walker P, Linehan J, Coffey C, Glasser B, Sheer L. Anxiety levels in women attending colposcopy clinics for treatment for cervical intraepithelial neoplasia: a randomized trial of written and video information. BJOG 2001;108:482-4.
8. Brooks SE, Gordon NJ, Keller SJ, Thomas SK, Chen TC, Moses G. Association of knowledge, anxiety, and fear with adherence to follow up for colposcopy. J Lower Genital Tract Dis 2002;6:17-22.

9. Stewart DE, Lickrish GM, Sierra S, Parkin H. The effect of educational brochures on knowledge and emotional distress in women with abnormal Papanicolaou smears. Obstet Gynecol 1993;81:280-2.

10. Wilkinson C, Jones JM, McBride J. Anxiety caused by abnormal result of cervical smear test: a controlled trial. BMJ 1990;300:440.

11. Lauver DR, Baggot A, Kruse K. Women's experiences in coping with abnormal Papanicolaou results and follow-up colposcopy. J Obstet Gynecol Neonatal Nurs 1999;28:283-90.

12. Campion MJ, Brown JR, McCance DJ, et al. Psychosexual trauma of an abnormal cervical smear. Br J Obstet Gynaecol 1988;95:175-81.

13. Lerman C, Miller SM, Scarborough R, Hanjani P, Nolte S, Smith D. Adverse psychologic consequences of positive cytologic cervical screening. Am J Obstet Gynecol 1991;165:658-62.

14. Jones MH, Singer A, Jenkins D. The mildly abnormal cervical smear: patient anxiety and choice management. J R Soc Med 1996;89:257-60.

15. Marteau TM, Walker P, Giles J, Smail M. Anxieties in women undergoing colposcopy. Br J Obstet Gynaecol 1990;97:859-61.

16. Roberts RA, Blunt SM. The psychological reaction of women to a colposcopy clinic. Br J Obstet Gynaecol 1994;101:751-2.

17. Gath DK, Hallam N, Mynors-Wallis L, Day A, Bond SAK. Emotional reactions in women attending a UK colposcopy clinic. J Epidemiol Community Health 1995;49:79-83.

18. Sharp LK, Lipsky MS. Screening for depression across the lifespan: a review of measures for use in primary care settings. Am Fam Physician 2002;66: 1001-8.

19. Ferris DG, Gilman PA, Lopez AG, Litaker MS, Miller JA, Macfee MS. The psychological effects women experience before and after a colposcopic examination and primary care appointment. J Lower Genital Tract Dis 2003;7:89-94.

20. Spitzer RL, Kroenke K, Williams JB. Validation and utility of a self-report version of PRIME-MD: the PHQ primary care study. Primary Care Evaluation of Mental Disorders. Patient Health Questionnaire. JAMA 1999;282:1737-44.

21. Fisher J, Corcoran K. Measures for clinical practice. A sourcebook. Vol 2, 2nd ed. New York: Maxwell Macmillan International, 1994:114-5.

22. Miller SM. When is a little information a dangerous thing?: Coping with stressful life events by monitoring vs. blunting. In: Levins S, Ursin H, eds. Coping and health. Proceedings of the 1979 NATO Inter- 
national Workshop on Coping and Health. New York: Plenum Press, 1980:145-69.

23. Miller SM, Roussi P, Altman D, Helm W, Steinberg A. Effects of coping style on psychological reactions of low income, minority women to colposcopy. J Reprod Med 1994;39:711-8.
24. Field MJ, Grigsby J. Telemedicine and remote patient monitoring. JAMA 2002;288:423-5.

25. Ferris DG, Litaker MS, Miller JA, Macfee MS, Crawley D, Watson D. Qualitative assessment of telemedicine network and computer-based telecolposcopy. J Lower Genital Tract Dis 2002;6:145-9. 